\title{
THIN METALLIC AND COMPOSITE PLATES EXPERIENCING LARGE DEFLECTIONS ABOVE THE VON KARMAN LIMITS
}

\author{
Sergey Selyugin \\ Stade, Germany \\ e-mail: sergey.selyugin@t-online.de
}

\begin{abstract}
Thin elastic plates (metallic or composite) experiencing large deflections are considered. The deflections are much larger than the plate thickness. The geometrically nonlinear elasticity theory and the Kirchhoff assumptions are employed. Small elongations and shears are assumed. Following Novozhilov, the strain expressions are derived. Then, under a small in-plane rotation assumption and using the virtual work principle, the equilibrium equations and the boundary conditions are obtained. The equations/conditions become the known von Karman ones for the case of moderate deflections. The solutions of the obtained equations may be used as benchmarks for the nonlinear structural analysis (e.g., FEM) software in the case of large deflections.
\end{abstract}

Keywords: thin plate, metal, composite, large deflection, geometric nonlinearity

(C) Sergey Selyugin, 2021 


\section{Introduction}

In the last decades, many researchers explored the nonlinear behavior of thin flexible plates. As a rule, they use the theory described in (Washizu 1975, Reddy 2007). The theory employs the Kirchhoff assumptions and the von Karman approach.

It is known that the latter approach describes the so-called moderate deflections (see Novozhilov 1961, 2011). The deflections are supposed to be comparable to the plate thickness. Reddy, from his side, indicates that in the von Karman approach the plate out-of-plane rotations should not be greater than $15^{\circ}$.

On the other hand, there are cases of considerable or even large deflections (much larger than plate thickness), e.g., considerable post-buckling shown in (Selyugin 2021).

It is known that the software for nonlinear structural analysis (like FEM) gives good results at the beginning of the nonlinear structural behavior. But in case of considerable nonlinear behavior the FEM numerical results become more different from the real structural behavior (the difference is recognized by the FEM experts).

The present paper deals with the case of large deflections. The deflections created due to the loading are much larger than the plate thickness. The virtual work principle is used to derive the equilibrium equations and the boundary conditions. The obtained equations and the conditions may be used for benchmarking the FEM software (dedicated to the nonlinear structural behavior).

\section{Theoretical analysis}

A thin elastic plate (homogeneous or composite) of constant thickness $h$ is considered. The plate thickness is much smaller than the linear plate dimensions. Fig. 1 demonstrates the coordinate system and some notations. The X-Y plane coincides with the mid-plane $\Gamma$ of the plate. The plate lateral surface $S$ consists of a loaded part $S_{1}$ and the part with the prescribed displacements $S_{2}$. The mid-plane $\Gamma$ intersects the lateral surface $S$ at the smooth contour $C$, with the portion $C_{1}$ belonging to $S_{1}$ and the portion $C_{2}$ belonging to $S_{2}$. The normal to $C$ is the vector $\vec{n}$ with the components $l, m$. The tangential direction to $C$ is $s$. The $n, s, z$ directions create the right triplet of vectors. The points of non-smoothness at the contour $C$ may be considered in an ordinary way, this will be done in a separate paper.

The plate experiences large deflections created due to loading (they are much larger than the plate thickness).

The geometrically nonlinear elasticity theory and the Kirchhoff assumptions are employed (Novozhilov 1961, 2011). The latter assumptions are written as follows:

$$
\varepsilon_{z z}=\varepsilon_{x z}=\varepsilon_{y z}=0
$$

where $\varepsilon$ is the Green strain tensor.

The deformed plate is supposed to be stable. 


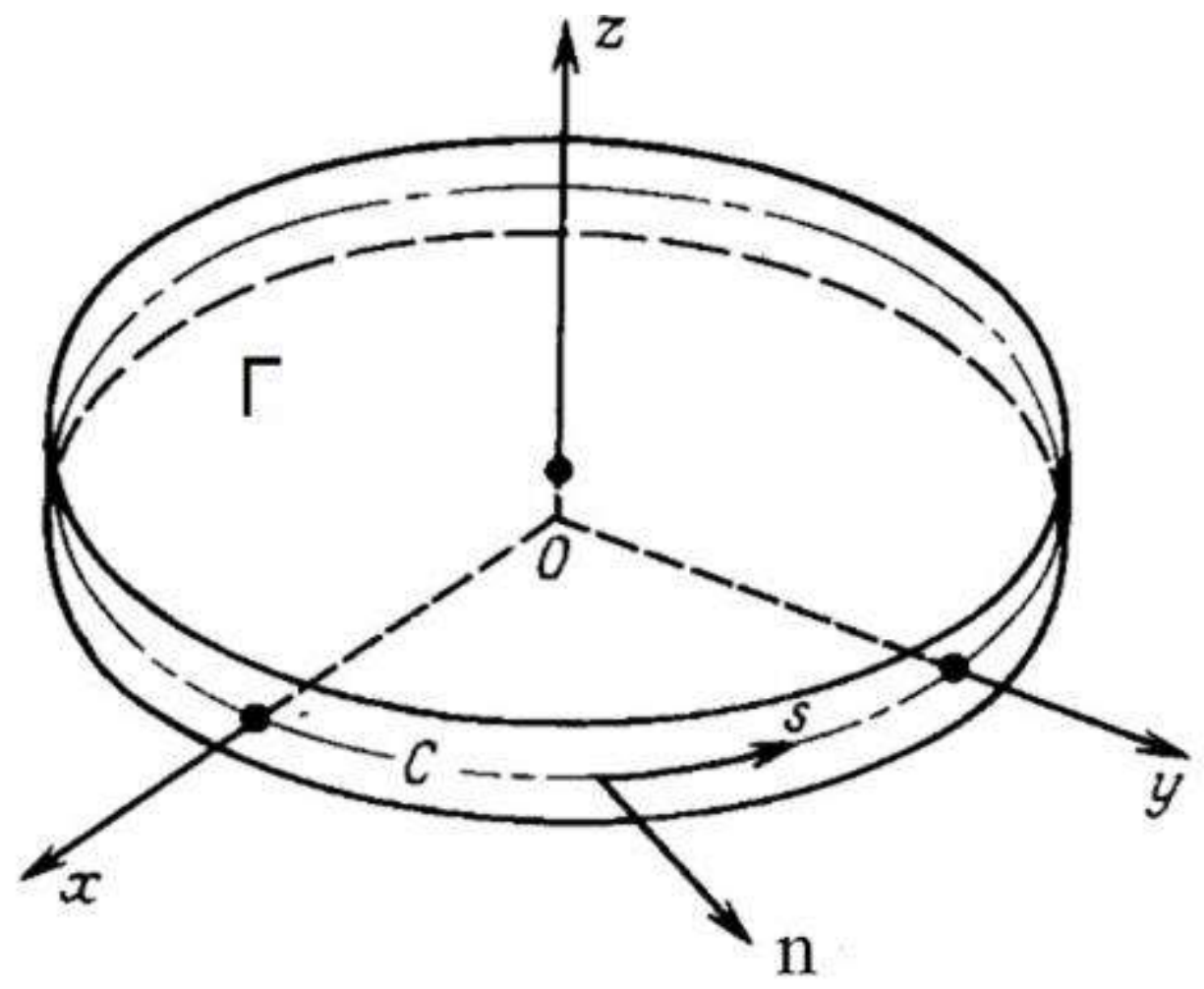

Fig. 1.

The ordinary notations are used hereinafter

$$
\begin{aligned}
& \frac{\partial}{\partial x}(\ldots)=(\ldots),{ }_{x} \\
& \frac{\partial}{\partial y}(\ldots)=(\ldots),{ }_{y}
\end{aligned}
$$

Small elongations and shears (negligible as compared to unity) are assumed. Following (Novozhilov, 1961, 2011), the displacements $u, v, w$ within the plate (respectively along X, Y, Z) are supposed to be

$$
\begin{aligned}
& u(x, y)=\hat{u}(x, y)+z \cdot \theta(x, y) \\
& \mathrm{v}(x, y)=\hat{\mathrm{v}}(x, y)+z \cdot \psi(x, y) \\
& w(x, y)=\hat{w}(x, y)+z \cdot \chi(x, y)
\end{aligned}
$$

where upper 'tilde' means quantities at the mid-plane. After using (3) and the definition of the Green tensor components it is obtained in (Novozhilov, 1961, 2011) that

$$
\begin{aligned}
& \theta=-\hat{w},_{x}\left(1+\hat{\mathrm{v}},{ }_{y}\right)+\hat{\mathrm{v}},{ }_{x} \hat{w},{ }_{y} \\
& \psi=-\hat{w},{ }_{y}\left(1+\hat{u},{ }_{x}\right)+\hat{u},{ }_{y} \hat{w},{ }_{x} \\
& \chi=\hat{u},{ }_{x}+\hat{\mathrm{v}},{ }_{y}+\hat{u},{ }_{x} \hat{\mathrm{v}},{ }_{y}-\hat{u},{ }_{y} \hat{\mathrm{v}},{ }_{x}
\end{aligned}
$$

and 


$$
\begin{aligned}
& \varepsilon_{x x}=\hat{\varepsilon}_{x x}+z \kappa_{x x}+z^{2} \eta_{x x} \\
& \varepsilon_{y y}=\hat{\varepsilon}_{y y}+z \kappa_{y y}+z^{2} \eta_{y y} \\
& \varepsilon_{x y}=\hat{\varepsilon}_{x y}+z \kappa_{x y}+z^{2} \eta_{x y}
\end{aligned}
$$

The mid-plane strains in (5) are

$$
\begin{aligned}
& \hat{\varepsilon}_{x x}=\hat{u},{ }_{x}+1 / 2\left[\hat{u}_{x}{ }_{x}+\hat{\mathrm{v}},{ }_{x}{ }_{x}+\hat{w}_{x_{x}}{ }^{2}\right] \\
& \hat{\varepsilon}_{y y}=\hat{\mathrm{v}},_{y}+1 / 2\left[\hat{u},{ }_{y}^{2}+\hat{\mathrm{v}}_{y}^{2}+\hat{w},{ }_{y}^{2}\right] \\
& 2 \hat{\varepsilon}_{x y}=\hat{u},_{y}+\hat{\mathrm{v}},_{x}+\hat{u},{ }_{y} \hat{u},{ }_{x}+\hat{\mathrm{v}},{ }_{x} \hat{\mathrm{v}},{ }_{y}+\hat{w},{ }_{x} \hat{w}_{y}
\end{aligned}
$$

and other quantities in (5):

$$
\begin{aligned}
& \kappa_{x x}=\theta,_{x}+\hat{u},{ }_{x} \theta,,_{x}+\hat{\mathrm{v}},{ }_{x} \psi,{ }_{x}+\hat{w},{ }_{x} \chi,_{x} \\
& \kappa_{y y}=\psi,{ }_{y}+\hat{u},{ }_{y} \theta,,_{y}+\hat{\mathrm{v}},{ }_{y} \psi,,_{y}+\hat{w},_{y} \chi,_{y} \\
& 2 \kappa_{x y}=\theta,_{y}+\psi,{ }_{x}+\hat{u},{ }_{x} \theta,,_{y}+\hat{u},{ }_{y} \theta,_{x}+\hat{\mathrm{v}},{ }_{x} \psi,{ }_{y}+\hat{\mathrm{v}},{ }_{y} \psi,{ }_{x}+\hat{w},{ }_{x} \chi,_{y}+\hat{w},{ }_{y} \chi,_{x} \\
& \eta_{x x}=1 / 2\left[\theta,{ }_{x}^{2}+\psi,{ }_{x}^{2}+\chi,{ }_{x}^{2}\right] \\
& \eta_{y y}=1 / 2\left[\theta,{ }_{y}^{2}+\psi,{ }_{y}^{2}+\chi,{ }_{y}^{2}\right] \\
& 2 \eta_{x y}=\theta,{ }_{x} \theta,{ }_{y}+\psi,{ }_{x} \psi,{ }_{y}+\chi,{ }_{x} \chi,{ }_{y}
\end{aligned}
$$

In (Novozhilov, 1961, 2011) it is indicated that for small elongations and shears the last (quadratic wrt to $z$ ) terms in (5) may be neglected. Then (5) is transformed to

$$
\begin{aligned}
& \varepsilon_{x x}=\hat{\varepsilon}_{x x}+z \kappa_{x x} \\
& \varepsilon_{y y}=\hat{\varepsilon}_{y y}+z \kappa_{y y} \\
& \varepsilon_{x y}=\hat{\varepsilon}_{x y}+z \kappa_{x y}
\end{aligned}
$$

Substituting (4) in (7), after cumbersome transformations we obtain

$$
\begin{aligned}
& \kappa_{x x}=-\theta(x, y) \hat{u},_{x x}-\psi(x, y) \hat{\mathrm{v}},_{x x}-(1+\chi(x, y)) \hat{w},_{x x} \\
& \kappa_{y y}=-\theta(x, y) \hat{u},_{y y}-\psi(x, y) \hat{\mathrm{v}},_{y y}-(1+\chi(x, y)) \hat{w},_{y y} \\
& \kappa_{x y}=-\theta(x, y) \hat{u},_{x y}-\psi(x, y) \hat{\mathrm{v}},_{x y}-(1+\chi(x, y)) \hat{w},_{x y}
\end{aligned}
$$

Further the in-plane rotations are supposed to be small. We have (see Novozhilov 1961, 2011)

where the in-plane rotation is

$$
e_{z z}=w,_{z z} \sim \varphi_{z}^{2} \approx \omega_{z}^{2}
$$

$$
\omega_{z}=1 / 2\left(\hat{u},{ }_{y}-\hat{\mathrm{v}},{ }_{x}\right)
$$

and $e_{z z}$ is the $z z$ component of the infinitesimal strain. Hence

$$
\chi \sim \varphi_{z}^{2}
$$

and in the case of small in-plane rotations (negligible as compared to unity)

$$
|\chi|<<1
$$

This means that (10) may be rewritten as 


$$
\begin{aligned}
& \kappa_{x x}=-\theta(x, y) \hat{u},_{x x}-\psi(x, y) \hat{\mathrm{v}},_{x x}-\hat{w},_{x x} \\
& \kappa_{y y}=-\theta(x, y) \hat{u},_{y y}-\psi(x, y) \hat{\mathrm{v}},_{y y}-\hat{w},_{y y} \\
& \kappa_{x y}=-\theta(x, y) \hat{u},_{x y}-\psi(x, y) \hat{\mathrm{v}},_{x y}-\hat{w},_{x y}
\end{aligned}
$$

The next step of our analysis is to use the virtual work principle (Washizu 1975). It is written as follows

$$
\int_{V} d V\left[\sigma_{x x} \delta \varepsilon_{x x}+\sigma_{y y} \delta \varepsilon_{y y}+2 \sigma_{x y} \delta \varepsilon_{x y}\right]-\int_{\Gamma} q(x, y) \delta w d \Gamma-\int_{S_{1}}\left[f_{x} \delta u+f_{y} \delta v+f_{z} \delta w\right] d S=0
$$

where $q, f_{x}, f_{x}, f_{x}, \delta, V$ respectively are the transverse external force per unit area applied at the surface $\Gamma$ of the plate, the X-Y-Z forces per unit area applied at the part $S_{1}$ of the plate lateral surface $S$, the variation symbol, the volume of the plate.

We introduce the following notations:

$$
\begin{array}{llll}
N_{x}=\int_{-h / 2}^{h / 2} \sigma_{x x} d z, & M_{x}=\int_{-h / 2}^{h / 2} z \sigma_{x x} d z, & F_{x}=\int_{-h / 2}^{h / 2} f_{x} d z, & \tilde{M}_{x}=\int_{-h / 2}^{h / 2} z f_{x} d z \\
N_{y}=\int_{-h / 2}^{h / 2} \sigma_{y y} d z, & M_{y}=\int_{-h / 2}^{h / 2} z \sigma_{y y} d z, & F_{y}=\int_{-h / 2}^{h / 2} f_{y} d z, & \tilde{M}_{y}=\int_{-h / 2}^{h / 2} z f_{y} d z \\
N_{x y}=\int_{-h / 2}^{h / 2} \sigma_{x y} d z, & M_{x y}=\int_{-h / 2}^{h / 2} z \sigma_{x y} d z & F_{z}=\int_{-h / 2}^{h / 2} f_{z} d z & \widetilde{F}_{z}=\int_{-h / 2}^{h / 2} z f_{z} d z
\end{array}
$$

Introducing (4), (6), (7), (9), (15), (17) in (16) and making necessary transformations, we obtain

$$
\int_{\Gamma} d \Gamma\left[\frac{\partial P_{x}}{\partial x}+\frac{\partial P_{y}}{\partial y}+K_{u} \delta \hat{u}+K_{v} \delta \hat{v}+K_{w} \delta \hat{w}\right]-\int_{\Gamma} q(x, y) \delta w d \Gamma-\int_{S_{1}}\left[f_{x} \delta u+f_{y} \delta v+f_{z} \delta w\right] d S=0
$$

where 


$$
\begin{aligned}
& K_{u}=-N_{x, x}-N_{x y, y}-\left(N_{x} \hat{u},_{x}+N_{x y} \hat{u},_{y}\right),_{x}-\left(N_{y} \hat{u},_{y}+N_{x y} \hat{u},{ }_{x}\right)_{y}-\left(M_{x} \theta\right),_{x x}-\left(M_{y} \theta\right)_{y y}- \\
& -2\left(M_{x y} \theta\right),{ }_{x y}+\Omega,{ }_{x} \hat{w},{ }_{y}-\Omega,{ }_{y} \hat{w},_{x} \\
& K_{v}=-N_{y, y}-N_{x y, x}-\left(N_{x} \hat{v},_{x}+N_{x y} \hat{v},_{y}\right),_{x}-\left(N_{y} \hat{v}_{y}+N_{x y} \hat{v},_{x}\right),_{y}-\left(M_{x} \psi\right),_{x x}-\left(M_{y} \psi\right),_{y y}- \\
& -2\left(M_{x y} \psi\right)_{x y}+R,{ }_{y} \hat{w}_{x}-R,{ }_{x} \hat{w}_{y} \\
& K_{w}=-M_{x, x x}-M_{y, y y}-2 M_{x y, x y}-\left(N_{x} \hat{w},_{x}+N_{x y} \hat{w},_{y}\right)_{x}-\left(N_{y} \hat{w},_{y}+N_{x y} \hat{w},_{x}\right),_{y}+R,,_{x}\left(1+\hat{u},{ }_{x}\right)- \\
& -R,{ }_{y}{ }_{,}+\Omega,{ }_{y}\left(1+\hat{u},{ }_{x}\right)-\Omega,{ }_{x} \hat{u},{ }_{y} \\
& P_{x}=\left[N_{x}\left(1+\hat{u},{ }_{x}\right)+N_{x y} \hat{u},{ }_{y}+\left(M_{x} \theta\right),_{x}+\left(M_{x y} \theta\right),{ }_{y}-\Omega \hat{w},{ }_{y}\right] \delta \hat{u}+ \\
& +\left[N_{x y}\left(1+\hat{v},{ }_{y}\right)+N_{x} \hat{v},_{x}+\left(M_{x} \psi\right),_{x}+\left(M_{x y} \psi\right),_{y}+R \hat{w},{ }_{y}\right] \delta \hat{v}+ \\
& +\left[N_{x y} \hat{w},{ }_{y}+N_{x} \hat{w},_{x}+M_{x, x}+M_{x y, y}-R\left(1+\hat{v},{ }_{y}\right)+\Omega \hat{u},{ }_{y}\right] \delta \hat{w}- \\
& -\left(M_{x} \theta\right) \delta \hat{u},{ }_{x}-\left(M_{x} \psi\right) \delta \hat{v},{ }_{x}-M_{x} \delta \hat{w},{ }_{x}-M_{x y} \theta \delta \hat{u},{ }_{y}-M_{x y} \psi \delta \hat{v},{ }_{y}-M_{x y} \delta \hat{w},{ }_{y} \\
& P_{y}=\left[N_{x y}\left(1+\hat{u},{ }_{x}\right)+N_{y} \hat{u},{ }_{y}+\left(M_{x y} \theta\right),_{x}+\left(M_{y} \theta\right)_{y}+\Omega \hat{w},_{x}\right] \delta \hat{u}+ \\
& +\left[N_{y}\left(1+\hat{v},{ }_{y}\right)+N_{x y} \hat{v},_{x}+\left(M_{x y} \psi\right),_{x}+\left(M_{y} \psi\right),_{y}-R \hat{w},_{x}\right] \delta \hat{v}+ \\
& +\left[N_{y} \hat{w},_{y}+N_{x y} \hat{w},_{x}+M_{y, y}+M_{x y, x}+R \hat{v},_{x}-\Omega\left(1+u,{ }_{x}\right)\right] \delta \hat{w}- \\
& -M_{x y} \theta \delta \hat{u},_{x}-M_{x y} \psi \delta \hat{v},_{x}-M_{x y} \delta \hat{w},_{x}-M_{y} \theta \delta \hat{u},{ }_{y}-M_{y} \psi \delta \hat{v},{ }_{y}-M_{y} \delta \hat{w},_{y} \\
& \Omega=-M_{x} \hat{v},_{x x}-M_{y} \hat{v},_{y y}-2 M_{x y} \hat{v},_{x y} \\
& R=-M_{x} \hat{u},_{x x}-M_{y} \hat{u},_{y y}-2 M_{x y} \hat{u},_{x y}
\end{aligned}
$$

Equilibrium equations for $x, y, z$ are respectively written as

$$
\begin{aligned}
& K_{u}=0 \\
& K_{v}=0 \\
& K_{w}-q=0
\end{aligned}
$$

The boundary conditions follow from the Gauss theorem:

$$
\int_{\Gamma}\left(P_{x, x}+P_{y, y}\right) d \Gamma=\int_{C}\left(\left(\begin{array}{c}
P_{x} \\
P_{y}
\end{array}\right)^{T}, \vec{n}\right) d C
$$

where $T$ means transposition. Equating to zero the multipliers to the variations of the independent variables we obtain the required stationarity conditions for the virtual work.

We indicate some relations to be used for the contour terms $(a, b, d$ are some functions of $x, y)$ :

$$
\begin{aligned}
& \frac{\partial}{\partial x}=l \frac{\partial}{\partial n}-m \frac{\partial}{\partial s} \\
& \frac{\partial}{\partial y}=m \frac{\partial}{\partial n}+l \frac{\partial}{\partial s} \\
& a d,,_{x}+b d,,_{y}=(a l+b m) d,,_{n}+(-a m+b l) d,,_{s}
\end{aligned}
$$

Continuing (22), we obtain: 


$$
\begin{aligned}
& \int_{\Gamma}\left(P_{x, x}+P_{y, y}\right) d \Gamma=\int_{C}\left(l P_{x}+m P_{y}\right) d C=\int_{C} d C\left\{\delta \hat { u } \left[\left(1+\hat{u},{ }_{x}\right)\left(N_{x} l+N_{x y} m\right)+\hat{u},{ }_{y}\left(N_{x y} l+N_{y} m\right)+\right.\right. \\
& \left.\left.+\Omega\left(-l \hat{w},{ }_{y}+m \hat{w},{ }_{x}\right)+l\left(\left(M_{x} \theta\right),_{x}+\left(M_{x y} \theta\right),_{y}\right)+m\left(\left(M_{x y} \theta\right),_{x}+\left(M_{y} \theta\right)\right)_{y}\right)\right]+\delta \hat{v}\left[\hat{v}_{x}\left(l N_{x}+m N_{x y}\right)+\right. \\
& \left.+\left(1+\hat{v},{ }_{y}\right)\left(l N_{x y}+m N_{y}\right)+R\left(l \hat{w},{ }_{y}-m \hat{w},_{x}\right)+l\left(\left(M_{x} \psi\right),_{x}+\left(M_{x y} \psi\right), y_{y}\right)+m\left(\left(M_{x y} \psi\right), x_{x}+\left(M_{y} \psi\right), y_{y}\right)\right]+ \\
& +\delta \hat{w}\left[l\left(N_{x} \hat{w},_{x}+N_{x y} \hat{w},_{y}+M_{x, x}+M_{x y, y}-R\left(1+\hat{v},_{y}\right)+\Omega \hat{u},_{y}\right)+m\left(N_{x y} \hat{w},_{x}+N_{y} \hat{w},{ }_{y}+M_{x y, x}+M_{y, y}+\right.\right. \\
& \left.\left.+R \hat{v},_{x}-\Omega\left(1+\hat{u},{ }_{x}\right)\right)\right]-\left(l^{2} M_{x} \theta+2 \operatorname{lm} M_{x y} \theta+m^{2} M_{y} \theta\right) \delta \hat{u},_{n}-\left(-\operatorname{lm}\left(M_{x}-M_{y}\right) \theta+\left(l^{2}-m^{2}\right) M_{x y} \theta\right) \delta \hat{u},_{s}- \\
& -\left(l^{2} M_{x} \psi+2 \operatorname{lm} M_{x y} \psi+m^{2} M_{y} \psi\right) \delta \hat{v}_{n}-\left(-\operatorname{lm}\left(M_{x}-M_{y}\right) \psi+\left(l^{2}-m^{2}\right) M_{x y} \psi\right) \delta \hat{v}_{s}- \\
& \left.-\left(l^{2} M_{x}+2 \operatorname{lm} M_{x y}+m^{2} M_{y}\right) \delta \hat{w}_{n}-\left(-\operatorname{lm}\left(M_{x}-M_{y}\right)+\left(l^{2}-m^{2}\right) M_{x y}\right) \delta \hat{w},_{s}\right\}
\end{aligned}
$$

The last integral in (18) may be transformed as follows:

$$
\begin{aligned}
& \int_{S_{1}}\left[f_{x} \delta u+f_{y} \delta v+f_{z} \delta w\right] d S=\int_{S_{1}} d S\left\{f_{x} \delta(\hat{u}-z \theta)+f_{y} \delta(\hat{v}-z \psi)+f_{z} \delta(\hat{w}-z \chi)\right\}= \\
& =\int_{C_{1}} d C\left\{F_{x} \delta \hat{u}+F_{y} \delta \hat{v}+F_{z} \delta \hat{w}+\tilde{M}_{x}\left[-\left(1+\hat{v},{ }_{y}\right) \delta \hat{w},_{x}-\hat{w},_{x} \delta \hat{v},_{y}+\hat{v},_{x} \delta \hat{w},_{y}+\hat{w},{ }_{y} \delta \hat{v},_{x}\right]+\right. \\
& +\tilde{M}_{y}\left[-\left(1+\hat{u},_{x}\right) \delta \hat{w},_{y}-\hat{w},_{y} \delta \hat{u},_{x}+\hat{u},{ }_{y} \delta \hat{w},_{x}+\hat{w},_{x} \delta \hat{u},,_{y}\right]+\widetilde{F}_{z}\left[\delta \hat{u},_{x}+\delta \hat{v},{ }_{y}+\hat{u},_{x} \delta \hat{v},_{y}+\hat{v},_{y} \delta \hat{u},_{x}-\right. \\
& \left.\left.-\hat{u},_{y} \delta \hat{v},_{x}-\hat{v},_{x} \delta \hat{u},{ }_{y}\right]\right\}=\int_{C_{1}} d C\left\{F_{x} \delta \hat{u}+F_{y} \delta \hat{v}+F_{z} \delta \hat{w}+\delta \hat{u},_{x}\left[-\tilde{M}_{y} \hat{w},_{y}+\widetilde{F}_{z}\left(1+\hat{v}_{y}\right)\right]+\right. \\
& +\delta \hat{u},{ }_{y}\left[\tilde{M}_{y} \hat{w},_{x}-\widetilde{F}_{z} \hat{v}_{x}\right]+\delta \hat{v},{ }_{x}\left[\tilde{M}_{x} \hat{w},{ }_{y}-\widetilde{F}_{z} \hat{u},{ }_{y}\right]+\delta \hat{v},{ }_{y}\left[-\tilde{M}_{x} \hat{w},_{x}+\widetilde{F}_{z}\left(1+\hat{u},_{x}\right)\right]+ \\
& +\delta \hat{w},_{x}\left[-\tilde{M}_{x}\left(1+\hat{v},{ }_{y}\right)+\tilde{M}_{y} \hat{u},{ }_{y}\right]+\delta \hat{w},{ }_{y}\left[\tilde{M}_{x} \hat{v}_{,_{x}}-\tilde{M}_{y}\left(1+\hat{u},{ }_{x}\right)\right]= \\
& =\int_{C_{1}} d C\left\{F_{x} \delta \hat{u}+F_{y} \delta \hat{v}+F_{z} \delta \hat{w}+\left[\left(-\tilde{M}_{y} \hat{w},_{y}+\widetilde{F}_{z}\left(1+\hat{v}_{,_{y}}\right)\right) l+\left(\tilde{M}_{y} \hat{w},_{x}-\widetilde{F}_{z} \hat{v},_{x}\right) m\right] \delta \hat{u},{ }_{n}+\right. \\
& +\left[-\left(-\widetilde{M}_{y} \hat{w},_{y}+\widetilde{F}_{z}\left(1+\hat{v}_{y}\right)\right) m+\left(\widetilde{M}_{y} \hat{w},_{x}-\widetilde{F}_{z} \hat{v},{ }_{x}\right)\right] \delta \hat{u},_{s}+ \\
& +\left[\left(\widetilde{M}_{x} \hat{w},{ }_{y}-\widetilde{F}_{z} \hat{u},{ }_{y}\right) l+\left(-\tilde{M}_{x} \hat{w},_{x}+\widetilde{F}_{z}\left(1+\hat{u},{ }_{x}\right)\right) m\right] \delta \hat{v}_{n}+ \\
& +\left[-\left(\widetilde{M}_{x} \hat{w},{ }_{y}-\widetilde{F}_{z} \hat{u},{ }_{y}\right) m+\left(-\widetilde{M}_{x} \hat{w},_{x}+\widetilde{F}_{z}\left(1+\hat{u},{ }_{x}\right)\right) z\right] \delta \hat{v}_{,_{s}}+ \\
& +\left[\left(-\tilde{M}_{x}\left(1+\hat{v}_{y}\right)+\tilde{M}_{y} \hat{u},{ }_{y}\right) l+\left(\tilde{M}_{x} \hat{v}_{{ }_{x}}-\tilde{M}_{y}\left(1+\hat{u},{ }_{x}\right)\right) m\right] \delta \hat{w},{ }_{n}+ \\
& \left.+\left[-\left(-\widetilde{M}_{x}\left(1+\hat{v},{ }_{y}\right)+\tilde{M}_{y} \hat{u},{ }_{y}\right) m+\left(\widetilde{M}_{x} \hat{v}_{x_{x}}-\widetilde{M}_{y}\left(1+\hat{u},_{x}\right)\right)\right] \delta \hat{w},_{s}\right\}
\end{aligned}
$$

Observing (18) - (24), we obtain 


$$
\begin{aligned}
& \int_{\Gamma}\left(P_{x, x}+P_{y, y}\right) d \Gamma-\int_{S_{1}}\left[f_{x} \delta u+f_{y} \delta v+f_{z} \delta w\right] d S= \\
& \int_{C} d C\left\{\delta \hat { u } \left[\left(1+\hat{u},{ }_{x}\right)\left(N_{x} l+N_{x y} m\right)+\hat{u},{ }_{y}\left(N_{x y} l+N_{y} m\right)+\Omega\left(-l \hat{w},{ }_{y}+m \hat{w},{ }_{x}\right)+\right.\right. \\
& \left.+l\left(\left(M_{x} \theta\right),_{x}+\left(M_{x y} \theta\right),{ }_{y}\right)+m\left(\left(M_{x y} \theta\right),_{x}+\left(M_{y} \theta\right),{ }_{y}\right)\right]+ \\
& +\delta \hat{v}\left[\hat{v},_{x}\left(l N_{x}+m N_{x y}\right)+\left(1+\hat{v},_{y}\right)\left(l N_{x y}+m N_{y}\right)+R\left(l \hat{w},{ }_{y}-m \hat{w},_{x}\right)+l\left(\left(M_{x} \psi\right),_{x}+\left(M_{x y} \psi\right),_{y}\right)+\right. \\
& \left.+m\left(\left(M_{x y} \psi\right),_{x}+\left(M_{y} \psi\right),{ }_{y}\right)\right]+ \\
& +\delta \hat{w}\left[l\left(N_{x} \hat{w},_{x}+N_{x y} \hat{w},_{y}+M_{x, x}+M_{x y, y}-R\left(1+\hat{v},_{y}\right)+\Omega \hat{u},_{y}\right)+m\left(N_{x y} \hat{w},_{x}+N_{y} \hat{w}_{,_{y}}+M_{x y, x}+M_{y, y}+\right.\right. \\
& \left.\left.+R \hat{v},{ }_{x}-\Omega\left(1+\hat{u},{ }_{x}\right)\right)\right]- \\
& -\left(l^{2} M_{x} \theta+2 \operatorname{lm} M_{x y} \theta+m^{2} M_{y} \theta\right) \delta \hat{u},{ }_{n}-\left(-\operatorname{lm}\left(M_{x}-M_{y}\right) \theta+\left(l^{2}-m^{2}\right) M_{x y} \theta\right) \delta \hat{u},{ }_{s}- \\
& -\left(l^{2} M_{x} \psi+2 \operatorname{lm} M_{x y} \psi+m^{2} M_{y} \psi\right) \delta \hat{v}_{n}-\left(-\operatorname{lm}\left(M_{x}-M_{y}\right) \psi+\left(l^{2}-m^{2}\right) M_{x y} \psi\right) \delta \hat{v},_{s}- \\
& \left.-\left(l^{2} M_{x}+2 \operatorname{lm} M_{x y}+m^{2} M_{y}\right) \delta \hat{w},_{n}-\left(-\operatorname{lm}\left(M_{x}-M_{y}\right)+\left(l^{2}-m^{2}\right) M_{x y}\right) \delta \hat{w},_{s}\right\}- \\
& -\int_{C_{1}} d C\left\{F_{x} \delta \hat{u}+F_{y} \delta \hat{v}+F_{z} \delta \hat{w}+\left[\left(-\widetilde{M}_{y} \hat{w},_{y}+\widetilde{F}_{z}\left(1+\hat{v}_{, y}\right)\right) l+\left(\tilde{M}_{y} \hat{w},_{x}-\widetilde{F}_{z} \hat{v}_{x}\right) m\right] \delta \hat{u},_{n}+\right. \\
& +\left[-\left(-\widetilde{M}_{y} \hat{w},_{y}+\widetilde{F}_{z}\left(1+\hat{v}_{y}\right)\right) m+\left(\widetilde{M}_{y} \hat{w},_{x}-\widetilde{F}_{z} \hat{v},{ }_{x}\right)\right] \delta \hat{u},{ }_{s}+ \\
& +\left[\left(\widetilde{M}_{x} \hat{w},{ }_{y}-\widetilde{F}_{z} \hat{u},_{y}\right) l+\left(-\widetilde{M}_{x} \hat{w},_{x}+\widetilde{F}_{z}\left(1+\hat{u},{ }_{x}\right)\right) m\right] \delta \hat{v},_{n}+ \\
& +\left[-\left(\widetilde{M}_{x} \hat{w},_{y}-\widetilde{F}_{z} \hat{u},{ }_{y}\right) m+\left(-\widetilde{M}_{x} \hat{w},_{x}+\widetilde{F}_{z}\left(1+\hat{u},{ }_{x}\right)\right) l\right] \delta \hat{v}_{,_{s}}+ \\
& +\left[\left(-\tilde{M}_{x}\left(1+\hat{v}_{y}\right)+\tilde{M}_{y} \hat{u},{ }_{y}\right) l+\left(\widetilde{M}_{x} \hat{v},_{x}-\tilde{M}_{y}\left(1+\hat{u},{ }_{x}\right)\right) m\right] \delta \hat{w},_{n}+ \\
& \left.+\left[-\left(-\widetilde{M}_{x}\left(1+\hat{v},{ }_{y}\right)+\tilde{M}_{y} \hat{u},{ }_{y}\right) m+\left(\tilde{M}_{x} \hat{v}_{{ }_{x}}-\tilde{M}_{y}\left(1+\hat{u},_{x}\right)\right) l\right] \delta \hat{w},_{s}\right\}
\end{aligned}
$$

Following the regular procedure, we should exclude the variations of the tangential derivatives $\delta \hat{u},_{s}, \delta \hat{v},_{s}, \delta \hat{w},_{s}$ from (25). The way of doing that is described in (Washizu 1975), (Reddy 2007). Having the contour integral like below and integrating by parts, we write

$$
\int_{G} H \frac{\partial \delta \beta}{\partial s} d s=\left.(H \delta \beta)\right|_{G}-\int_{G} \delta \beta \frac{\partial H}{\partial s} d s
$$

where $H, \delta \beta$ are some functions at the contour $G$ and the first rhs term means the difference of the squared product value at the ends of the contour.

We introduce the notations:

$$
\begin{aligned}
& A_{u}=\left(\operatorname{lm}\left(M_{x}-M_{y}\right) \theta-\left(l^{2}-m^{2}\right) M_{x y} \theta\right)+\left[\left(-\widetilde{M}_{y} \hat{w},_{y}+\widetilde{F}_{z}\left(1+\hat{v},_{y}\right)\right) m-\left(\widetilde{M}_{y} \hat{w}_{x_{x}}-\widetilde{F}_{z} \hat{v}_{x}\right) l\right] \\
& A_{v}=\left(\operatorname{lm}\left(M_{x}-M_{y}\right) \psi-\left(l^{2}-m^{2}\right) M_{x y} \psi\right)+\left[\left(\widetilde{M}_{x} \hat{w},_{y}-\widetilde{F}_{z} \hat{u},{ }_{y}\right) m-\left(-\tilde{M}_{x} \hat{w}_{x_{x}}+\widetilde{F}_{z}\left(1+\hat{u},{ }_{x}\right)\right)\right] \\
& A_{w}=\left(\operatorname{lm}\left(M_{x}-M_{y}\right)-\left(l^{2}-m^{2}\right) M_{x y}\right)+\left[\left(-\tilde{M}_{x}\left(1+\hat{v}_{,}\right)+\tilde{M}_{y} \hat{u}_{y}\right) m-\left(\tilde{M}_{x} \hat{v}_{x}-\tilde{M}_{y}\left(1+\hat{u},{ }_{x}\right)\right)\right]
\end{aligned}
$$

Then all terms in (25) containing variations of the tangential derivatives of $\hat{u}, \hat{v}, \hat{w}$ are written as follows:

$$
A_{u} \delta \hat{u},{ }_{s}+A_{v} \delta \hat{v},{ }_{s}+A_{w} \delta \hat{w},{ }_{s}
$$

It should be noted that the square-bracketed terms are non-zero ones at the loaded part $C_{1}$ only. 
Transforming the contour integral of (31) in view of (26) and removing the terms at the ends of the contour (the variations of the displacements at the ends of the supported contour portion $C_{2}$ are equal to zero), we obtain the under-integral term, corresponding to the tangential derivatives of the displacements $\hat{u}, \hat{v}, \hat{w}$ as:

$$
\frac{\partial A_{u}}{\partial s} \delta \hat{u}+\frac{\partial A_{v}}{\partial s} \delta \hat{v}+\frac{\partial A_{w}}{\partial s} \delta \hat{w}
$$

It is clear now that (32) will give some extra terms for the boundary conditions. The developed relations lead to the boundary conditions. Namely, at $C_{1}$ the multipliers to $\delta \hat{u}, \delta \hat{v}, \delta \hat{w}, \delta \hat{u}_{n}, \delta \hat{v},{ }_{n}, \delta \hat{w},_{n}$ should be equal to zero. This gives us six relations at $C_{1}$ :

$$
\begin{aligned}
& \left\lfloor\left(1+\hat{u},,_{x}\right)\left(N_{x} l+N_{x y} m\right)+\hat{u}_{y}\left(N_{x y} l+N_{y} m\right)+\Omega\left(-l \hat{w},{ }_{y}+m \hat{w}_{,}\right)+\right. \\
& \left.+l\left(\left(M_{x} \theta\right)_{x_{x}}+\left(M_{x y} \theta\right)_{y}\right)+m\left(\left(M_{x y} \theta\right)_{x_{x}}+\left(M_{y} \theta\right),_{y}\right)\right]+\frac{\partial A_{u}}{\partial s}-F_{x}=0 \\
& {\left[\hat{v}_{x}\left(l N_{x}+m N_{x y}\right)+\left(1+\hat{v}_{y}\right)\left(l N_{x y}+m N_{y}\right)+R\left(l \hat{w},{ }_{y}-m \hat{w},_{x}\right)+\right.} \\
& \left.+l\left(\left(M_{x} \psi\right)_{x}+\left(M_{x y} \psi\right)_{y}\right)+m\left(\left(M_{x y} \psi\right)_{x}+\left(M_{y} \psi\right), y\right)\right]+\frac{\partial A_{v}}{\partial s}-F_{y}=0 \\
& \left\lfloor l\left(N_{x} \hat{w},_{x}+N_{x y} \hat{w},_{y}+M_{x, x}+M_{x y, y}-R\left(1+\hat{v}_{y}\right)+\Omega \hat{u}_{,}\right)-\right. \\
& \left.+m\left(N_{x y} \hat{w},{ }_{x}+N_{y} \hat{w}_{y}+M_{x y, x}+M_{y, y}+R \hat{v},{ }_{x}-\Omega\left(1+\hat{u},{ }_{x}\right)\right)\right]+\frac{\partial A_{w}}{\partial s}-F_{z}=0 \\
& -\left(l^{2} M_{x} \theta+2 l m M_{x y} \theta+m^{2} M_{y} \theta\right)-\left[\left(-\widetilde{M}_{y} \hat{w}_{y}+\widetilde{F}_{z}\left(1+\hat{v}_{y}\right)\right) l+\left(\widetilde{M}_{y} \hat{w}_{x_{x}}-\widetilde{F}_{z} \hat{v}_{x}\right) m\right]=0 \\
& -\left(l^{2} M_{x} \psi+2 \operatorname{lm} M_{x y} \psi+m^{2} M_{y} \psi\right)-\left[\left(\widetilde{M}_{x} \hat{w},_{y}-\widetilde{F}_{z} \hat{u}_{y}\right) l+\left(-\widetilde{M}_{x} \hat{w}_{x}+\widetilde{F}_{z}\left(1+\hat{u},_{x}\right)\right) m\right]=0 \\
& -\left(l^{2} M_{x}+2 l m M_{x y}+m^{2} M_{y}\right)-\left[\left(-\widetilde{M}_{x}\left(1+\hat{v}_{y}\right)+\widetilde{M}_{y} \hat{u}_{y}\right) l+\left(\widetilde{M}_{x} \hat{v}_{x}-\widetilde{M}_{y}(1+\hat{u}, x)\right) m\right]=0
\end{aligned}
$$

At $C_{2}$ we obtain the clamping conditions

$$
\delta \hat{u}=\delta \hat{v}=\delta \hat{w}=\delta \hat{u},{ }_{n}=\delta \hat{v},{ }_{n}=\delta \hat{w},{ }_{n}=0
$$

Other types of the boundary conditions should be considered in a similar way via analysis of the variational integral.

The equilibrium equations (20) should be complemented by the relations determining the forces and the moments via the mid-plane displacements and their derivatives. The author intends to describe the details of the subject in the following papers.

\section{Discussion}

Every equilibrium equation from (20) expressed in $\hat{u}, \hat{v}, \hat{w}$ has the order four. Hence, the order of the equilibrium equation system (20) is twelve. There are six unknown primary variables $\hat{u}, \hat{v}, \hat{w}, \hat{u}{ }_{n}, \hat{v},_{n}, \hat{w}_{{ }_{n}}$ to be determined from (20). We have six boundary conditions at every contour portion, namely the natural boundary conditions (25)-(30) at $C_{1}$ and the essential boundary conditions (31) at $C_{2}$. This means that the developed system of equations is consistent.

When we consider the moderate deflections only, it is easy to see that in the quantities $K_{u}, K_{v}, K_{w}$ respectively two, two and five initial terms (corresponding to the von Karman case) 
remain. In the boundary conditions the variables $\theta, \psi$ become equal to zero. The quantities $A_{u}, A_{v}$ disappear (or, in other words, equal to zero). The quantities $\hat{u},_{x}, \hat{u},{ }_{y}, \hat{v},_{x}, \hat{v},{ }_{y}$ are neglected and taken equal to zero. The conditions (25)-(27), (30) are transformed to the von Karman case (see Reddy 2007). The conditions (28), (29) disappear. The variables $\hat{u}, \hat{v}, \hat{u}{ }_{n}, \hat{v}_{{ }_{n}}$ leave the list of the primary ones.

\section{Conclusions}

- The equilibrium equations are derived.

- The obtained system of equilibrium equations is consistent

- There are six primary variables in the system, namely $\hat{u}, \hat{v}, \hat{w}, \hat{u},{ }_{n}, \hat{v}{ }_{n}, \hat{w},_{n}$

- The essential and natural boundary conditions are obtained.

- For moderate deflections the equilibrium equations and the boundary conditions are transformed to ones of the von Karman case

- The solutions of the derived equations may be used as benchmarks for the nonlinear structural analysis software like FEM.

\section{REFERENCES}

Novozhilov V., 2011. Foundations of the nonlinear theory of elasticity. Dover Publ., 256 pp.

Novozhilov V., 1961. Theory of elasticity. Pergamon Press, 448 pp.

Reddy J.N., 2007. Theory and analysis of elastic plates and shells. $2^{\text {nd }}$ edition. Taylor \& Francis Group, New York.

Selyugin S., 2021. Engineering stress and strength analysis of composite plates (using FEM and Classical Lamination Plate Theory CLPT). DOI: $\underline{10.13140 / \text { RG.2.2.14779.54568 }}$

Washizu K., 1975. Variational Methods in Elasticity and Plasticity. $2^{\text {nd }}$ edition, Pergamon Press. 412 pp. 